\title{
ON A THEOREM OF SZEGÖ
}

\section{HENRY HELSON ${ }^{1}$}

The subject of this note is an extension of the following well-known theorem of Szegö [8]: Suppose the number of distinct coefficients in a power series

$$
u(r, x)=\sum_{k=0}^{\infty} a_{k} r^{k} e^{i k x}
$$

is finite. Assume that the analytic function $u(r, x)$ can be continued across some arc of the boundary of the unit circle. Then the $a_{k}$ are equal, beyond some point, to the terms of a periodic sequence.

A number of generalizations and related results have been published $[2 ; 3 ; 4 ; 5 ; 9]$, of which we mention in particular that of Duffin and Schaeffer [4]; these authors replace the hypothesis that $u(r, x)$ is analytically continuable by the weaker assumption that the function is bounded in some sector of the circle. A theorem of the same type, but apparently not implied by the others, was proved in [5]: If the harmonic function

$$
u(r, x)=\sum_{k=-\infty}^{\infty} a_{k} r^{|k|} e^{i k x}
$$

has, as before, only finitely many distinct coefficients, and satisfies the growth condition

$$
\int_{0}^{2 \pi}|u(r, x)| d x \leqq M<\infty \quad(r<1),
$$

then the sequence $\left\{a_{k}\right\}$ coincides with some periodic sequence except in a finite set of indices.

The purpose of this paper is to give a common generalization of these theorems, exhibiting (we believe) the essential features of the problem and proved in a new way.

THEOREM. Let $u(r, x)=\sum_{k=-\infty}^{\infty} a_{k} r^{|k|} e^{i k x}$ have only finitely many distinct coefficients, and for some arc $(\alpha, \beta)$ of the circle satisfy

$$
\int_{\alpha}^{\beta}|u(r, x)| d x \leqq M<\infty \quad(r<1) .
$$

Received by the editors March 3, 1954.

1 Jewett Fellow of the Bell Telephone Laboratories. 
Then $\left\{a_{k}\right\}$ is ultimately periodic to the right, and ultimately periodic to the left.

As far as we have verified, our method of proof yields all those generalizations of Szegö's theorem in which the coefficients are supposed to be bounded. The theorem of the author referred to above is obtained by taking for $(\alpha, \beta)$ the entire circle. Then it is necessary to remark that if a sequence of Fourier-Stieltjes coefficients is periodic to the right and to the left, it must coincide except in a finite set of indices with a single periodic sequence.

The proof is based on two facts.

TheOREM OF F. AND M. RiEsz [6]. Let $\mu$ be a complex bounded Borel measure ${ }^{2}$ on the circle for which the Fourier-Stieltjes coefficients

$$
b_{n}=\frac{1}{2 \pi} \int_{0}^{2 \pi} e^{-i n x} d \mu(x)
$$

vanish for all sufficiently large positive (or all large negative) indices. Then $\mu$ is absolutely continuous with respect to Lebesgue measure. ${ }^{3}$

Lемма. Let $\left\{n_{k}\right\}$ be an increasing sequence of integers, and let $\mu$ and $\gamma$ be complex bounded measures on the circle. Suppose that

$$
\int_{0}^{2 \pi} \phi(x) d \gamma(x)=\lim _{k=\infty} \int_{0}^{2 \pi} e^{-i n_{k} x} \phi(x) d \mu(x)
$$

for every continuous function $\phi$ on the circle. Then $\gamma$ is singular ${ }^{4}$ with respect to Lebesgue measure. The same conclusion holds if for two increasing sequences $\left\{n_{k}\right\}$ and $\left\{m_{k}\right\}$ the measures satisfy

$$
\int_{0}^{2 \pi} \phi(x) d \gamma(x)=\lim _{k=\infty} \int_{0}^{2 \pi}\left(e^{-i n_{k} x}-e^{-i m_{k} x}\right) \phi(x) d \mu(x)
$$

for all continuous $\phi$.

The lemma seems to be new, and we shall sketch a proof. It is enough to consider the hypothesis in its first form, the second being quite analogous. For the absolutely continuous part $\mu_{a}$ of $\mu$, we have

2 The reader who speaks of functions of bounded variation rather than of complex measures will have no difficulty in interpreting our terminology.

3 A simple proof of the result in [5] can be given using this theorem. Conversely, the Riesz theorem can be proved using the main argument in [5]. We were unaware until recently how closely related that argument is to the one originally employed in the Riesz paper.

4 We mean that $\gamma$ has no absolutely continuous part, but it may contain point masses. 
by the Riemann-Lebesgue Theorem

$$
\lim _{k=\infty} \int_{0}^{2 \pi} e^{-i n_{k} x} \phi(x) d \mu_{a}(x)=0 .
$$

Consequently for the singular part $\mu_{s}$,

$$
\int_{0}^{2 \pi} \phi(x) d \gamma(x)=\lim _{k=\infty} \int_{0}^{2 \pi} e^{-i n_{k} x} \phi(x) d \mu_{s}(x) .
$$

Fix a point $x$ on the circle and a small positive number $\epsilon$. As $\phi$ ranges over the continuous functions vanishing outside the interval $(x-\epsilon$, $x+\epsilon)$ and bounded by one in absolute value, the supremum of

$$
\left|\int_{0}^{2 \pi} \phi(x) d \gamma(x)\right|
$$

is exactly the total variation of $\gamma$ over $(x-\epsilon, x+\epsilon)$. At the same time

$$
\lim _{k=\infty}\left|\int_{0}^{2 \pi} e^{-i n_{k} x} \phi(x) d \mu_{s}(x)\right|
$$

never exceeds the variation of $\mu_{s}$ over the same interval. Since $\mu_{s}$ is a singular measure, for almost every point $x$ this variation is $o(\epsilon)$ as $\epsilon$ tends to zero. Hence the variation of $\gamma$ is $o(\epsilon)$ for almost all $x$, and this is possible only if $\gamma$ is itself singular, as we had to show.

We proceed to the proof of the main theorem. Since only finitely many $a_{k}$ are distinct, for each positive integer $p$ there are arbitrarily large distinct indices $m$ and $n$ such that

$$
a_{m-1}=a_{n-1}, \cdots, a_{m-p}=a_{n-p} .
$$

If, contrary to the conclusion of the theorem, $\left\{a_{k}\right\}$ is not periodic to the right, we can choose $m$ and $n$ so that

$$
a_{m} \neq a_{n} .
$$

We choose and fix sequences $m(p)$ and $n(p)$ satisfying these conditions and tending to infinity with $p$.

For each $p$ define the new sequence

$$
b_{k}^{p}=a_{m+k}-a_{n+k} .
$$

Evidently the numbers $b_{k}^{p}$ assume only finitely many values, and so are bounded from zero when they do not vanish. Moreover

$$
b_{0}^{p} \neq 0, \quad b_{-1}^{p}=\cdots=b_{-p}^{p}=0 .
$$


By a diagonal process we can find a sequence of integers $p_{1}, p_{2}, \cdots$ tending to infinity such that the limit

$$
b_{k}=\lim _{j=\infty} b_{k}^{p_{j}}
$$

exists for each $k$. Clearly

$$
b_{0} \neq 0, \quad b_{k}=0 \quad(\text { all } k<0) .
$$

The sequence $\left\{b_{k}\right\}$ is the main object of study. ${ }^{5}$

Let $\epsilon$ be a positive number smaller than the length of the arc $(\alpha, \beta)$. Construct a triangular function equal to one at the origin, decreasing linearly to zero at the points $\pm \epsilon / 2$, vanishing elsewhere in $(-\pi, \pi)$, and periodic. Denote by $f$ the translate of the triangular function having its peak at the center of $(\alpha, \beta)$. By computation we can verify that its coefficients satisfy

$$
c_{k}=\frac{1}{2 \pi} \int_{0}^{2 \pi} f(x) e^{-i k x} d x=O\left(1 / k^{2}\right) .
$$

Form the convolution

$$
d_{k}=\sum_{n=0}^{\infty} b_{n} c_{k-n}
$$

we shall prove in several steps that all the $d_{k}$ vanish.

Leмма. The $d_{k}$ are the Fourier-Stieltjes coefficients of a bounded complex measure $\mu$ on the circle.

For $r<1$ form the continuous function

$$
u_{r}(x)=f(x) u(r, x) .
$$

Then

$$
\int_{0}^{2 \pi}|u(r, x) f(x)| d x \leqq \int_{\alpha}^{\beta}|u(r, x)| d x \leqq M,
$$

so the norms of the $u_{r}$ are bounded in $L(0,2 \pi)$. By the Helly theorem, there is a bounded complex measure $\nu$ on the circle and a sequence of values of $r$ increasing to one such that

$$
\int_{0}^{2 \pi} \phi(x) d \nu(x)=\lim \int_{0}^{2 \pi} u_{r}(x) \phi(x) d x
$$

- An argument involving translation of the coefficient sequence is used in the original proof of Szegö's theorem [1, pp. 315-319]. The weak limiting process, however, is new. 
for every continuous periodic function $\phi$. Choosing an exponential for $\phi$,

$$
\begin{aligned}
\frac{1}{2 \pi} \int_{0}^{2 \pi} e^{-i q x} d \nu(x) & =\frac{1}{2 \pi} \lim \int_{0}^{2 \pi} u(r, x) f(x) e^{-i q x} d x \\
& =\lim \sum_{k=-\infty}^{\infty} a_{k}{ }^{|k|} c_{q-k}=\sum_{k=-\infty}^{\infty} a_{k} c_{q-k} .
\end{aligned}
$$

Thus the Fourier-Stieltjes coefficients of $\nu$ are given by the convolution of the $a_{k}$ and the $c_{k}$. The $b_{k}$ were obtained from the $a_{k}$ by a process of translation and subtraction. Recalling the construction, which was carried through by means of sequences $m(p)$ and $n(p)$, it is easy to verify that the typical coefficient of the measure

$$
\left(e^{-i m x}-e^{-i n x}\right) d \nu(x)
$$

is given by the convolution

$$
\sum_{k=-\infty}^{\infty} b_{k}^{p} c_{q-k}
$$

As $p$ tends to infinity through the sequence of values $p_{1}, p_{2}, \cdots$ the sum tends to

$$
d_{q}=\sum_{k=0}^{\infty} b_{k} c_{q-k}
$$

But the total variation of the measures is uniformly bounded, and so by the Helly theorem again an appropriate subsequence of measures tends weakly to a measure $\mu$ whose coefficients are the $d_{q}$.

LEMMA. The measure $\mu$ is singular with respect to Lebesgue measure.

Indeed, $\mu$ is the weak limit of a sequence of measures of the form

$$
\left(e^{-i m x}-e^{-i n x}\right) d \nu(x)
$$

as $m$ and $n$ tend to infinity in a certain way. The first lemma asserts directly that $\mu$ is singular.

Lemma. The singular measure $\mu$ is also absolutely continuous.

If $K$ is a constant larger than any $\left|b_{k}\right|$, then

$$
\left|d_{q}\right| \leqq K \sum_{k=0}^{\infty}\left|c_{q-k}\right|=K \sum_{k=-\infty}^{q}\left|c_{k}\right| \text {. }
$$

We know that $c_{k}=O\left(1 / k^{2}\right)$, and hence for large negative $q$ 


$$
d_{q}=O(1 / q)
$$

Consequently

$$
\sum_{q=-\infty}^{0}\left|d_{q}\right|^{2}<\infty .
$$

It follows that

$$
\sum_{q=-\infty}^{0} d_{q} e^{i q x}
$$

is the Fourier series of a square-summable function, and a fortiori of an absolutely continuous measure. Hence the complementary sum

$$
\sum_{q=1}^{\infty} d_{q} e^{i q x}
$$

is a Fourier-Stieltjes series, by the Riesz theorem of an absolutely continuous measure. Thus the full series

$$
\sum_{q=-\infty}^{\infty} d_{q} e^{i q x}
$$

is the Fourier-Stieltjes series of an absolutely continuous measure, as we had to show.

A measure which is both singular and absolutely continuous is the zero measure, so the coefficients $d_{k}$ all vanish. That is, for all $q$

$$
\sum_{k=0}^{\infty} b_{k} c_{q-k}=0 .
$$

We want to conclude that all the $b_{k}$ are zero. ${ }^{6}$ Observe that if $x$ is sufficiently close to the origin, the numbers $c_{k} e^{i k x}$ are the Fourier coefficients of a translate of the triangular function $f$, which still vanishes on $(\alpha, \beta)$. Applying the preceding considerations anew, we have for each $q$

$$
\sum_{k=0}^{\infty} b_{k} c_{q-k} e^{i(q-k) x}=0
$$

or

$$
\sum_{k=0}^{\infty} b_{k} e^{-i k x} c_{q-k}=0
$$

- The argument from this point is intended to establish this fact as conveniently as possible. The conclusion is well known. It is nevertheless interesting that a direct proof, not involving analytic functions, can be given, based purely on the theory of linear spaces. 
More generally, if $h$ is any summable function vanishing outside a certain neighborhood of the origin,

$$
\sum_{k=0}^{\infty} b_{k} \int_{0}^{2 \pi} h(x) e^{-i k x} d x c_{q-k}=0 .
$$

Denoting the Fourier coefficients of $h$ by $e_{k}$,

$$
\sum_{k=0}^{\infty} b_{k} e_{k} c_{q-k}=0 \text {. }
$$

If we choose for $h$ a triangular function, then the sequence $\left\{b_{k} e_{k}\right\}$ is square-summable, and represents the Fourier coefficients of a squaresummable function $H$. The last convolution then expresses the fact that the product $f H$ vanishes identically. Since $f$ is a triangular function, $H$ must vanish on an arc of the circle. Because $H$ is the boundary function of an analytic function in the unit circle, $H$ must vanish almost everywhere. None of the coefficients $e_{k}$ is zero, and so the $b_{k}$ vanish, in particular $b_{0}$. But $b_{0}$ is known to be different from zero. The contradiction stems from the assumption that $\left\{a_{k}\right\}$ was not periodic to the right. Similarly we can show that $\left\{a_{k}\right\}$ is periodic to the left, and the proof of the theorem is complete.

A new situation arises when the assumption that the coefficients $a_{k}$ are bounded is replaced by some condition of arithmetic nature. The theorem of Carlson and Pólya $[3 ; 1]$ is of this type: if an analytic function in the circle has integral coefficients and can be analytically continued across some part of the boundary, then it is rational. Continuing work of Pisot and others, Salem [7] proves a number of theorems about power series with integral coefficients which exhibit clearly the algebraic nature of the problems. Moreover he gives an example of a power series with integral coefficients, analytic in the circle, and bounded in a sector, which is not a rational function. Thus no extension of the theorem of Carlson and Pólya analogous to the generalization we have found of Szegö's theorem can be true, in spite of the similarity in the statement of the two theorems.

\section{REFERENCES}

1. L. Bieberbach, Lehrbuch der Funktionentheorie, vol. 11, reprinted, New York, 1945.

2. F. Carlson, Über Potenzreihen mit endlich vielen verschiedenen Koeffizienten, Math. Ann. vol. 79 (1919) pp. 237-245.

3. - Über Potenzreihen mit ganzzahligen Koeffizienten, Math. Zeit. vol. 9 (1921) pp. 1-13.

4. R. J. Duffin and A. C. Schaeffer, Power series with bounded coefficients, Amer. J. Math. vol. 67 (1945) pp. 141-154. 
5. H. Helson, Note on harmonic functions, Proc. Amer. Math. Soc. vol. 4 (1953) pp. 686-691.

6. F. and M. Riesz, Über die Randwerte einer analytischen Funktion, Quatrième Congrès des Math. Scand., 1916, pp. 27-44.

7. R. Salem, Power series with integral coefficients, Duke Math. J. vol. 12 (1945) pp. 153-172.

8. G. Szegö, Über Potenzreihen mit endlich vielen verschiedenen Koeffizienten, Sitzungsgerichte der preussischen Akademie der Wissenschaften (1922) pp. 88-91.

9. - Tschebyscheffsche Polynome und nichtfortsetzbare Potenzreihen, Math. Ann. vol. 87 (1922) pp. 90-111.

Yale University 